\title{
A Single Centre Experience with Phenotype Dependent Perioperative Chemotherapy for Primary Breast Cancer
}

\author{
Tamás Kullmann*, Kofi Agyemang-Prempeh, Adél Ambrus, Gabriella Herodek, Balázs Hillier, \\ Károly Kocsis, Ilona Laszip, Mária Mák, Tamás Pintér, István Sipőcz \\ Department of Oncoradiology, Petz Aladár Hospital, Győr, Hungary \\ Email: *kullmanndoki@hotmail.com
}

How to cite this paper: Kullmann, T., Agyemang-Prempeh, K., Ambrus, A., Herodek, G., Hillier, B., Kocsis, K., Laszip, I., Mák, M., Pintér, T. and Sipőcz, I. (2019) A Single Centre Experience with Phenotype Dependent Perioperative Chemotherapy for Primary Breast Cancer. Journal of Cancer Therapy, 10, 21-27.

https://doi.org/10.4236/jct.2019.101002

Received: December 12, 2018

Accepted: January 1, 2019

Published: January 4, 2019

Copyright (@) 2019 by authors and Scientific Research Publishing Inc. This work is licensed under the Creative Commons Attribution International License (CC BY 4.0).

http://creativecommons.org/licenses/by/4.0/

\begin{abstract}
Background: Standard adjuvant or neo-adjuvant chemotherapy of primary breast cancers consists of docetaxel and the combination of anthracyclin-cyclophosphamide in two steps. According to international literature, the neo-adjuvant treatment of triple negative tumours by the combination of docetaxel-carboplatin allows a high rate of pathological complete remission and the adjuvant treatment of small, node negative HER2+ tumours by the combination of paclitaxel-trastuzumab allows a negligible recurrence rate together with a very good tolerance. Methods: Our aim was the retrospective analysis of the alternative chemotherapy regimens in our patient population. Results: The neo-adjuvant docetaxel-carboplatin allowed a 55\% pathological complete remission rate among 20 patients. With the adjuvant paclitaxel-trastuzumab, we did not notice any recurrence among 5 patients at a median follow-up of 18 months. Conclusion: Our results correspond to the literature data. The spread of the protocols in the clinical practice is proposed.
\end{abstract}

\section{Keywords}

Breast Cancer, Carboplatin, Trastuzumab, Adjuvant, Neoadjuvant

\section{Introduction}

Localised or regionally advanced invasive breast cancer is curable in $75 \%$ of the cases [1]. The orchestered administration of several anti-cancer modalities including surgery, radio- and chemotherapy, hormonal and targeted treatment is mandatory to reach such a high rate of cure. The so far obligatory element and reference point of the multimodal approach are the surgical removal of the pri- 
mary tumour [2]. The necessity of other interventions depends on the size, extension and receptor status of the tumour.

The principal aim of the chemotherapy is the eradication of the micro-metastases, present at the time of the diagnosis of the disease, even if undetectable by the staging examinations with other words the lowering of the risk of distant recurrence. Chemotherapy administered after surgery is called adjuvant that before surgery is called neo-adjuvant. In presence of palpable axillary lymph nodes the first element of the multimodal treatment is the neo-adjuvant chemotherapy. In absence of palpable axillary lymph nodes the order of surgery and chemotherapy may be determined relatively freely, taking into account several arguments. It has not been proven that either the adjuvant or the neo-adjuvant application of the chemotherapy could provide any advantage over the other in terms of overall survival [3].

In case of a proportionately big primary tumour the neo-adjuvant chemotherapy may allow a breast conserving intervention, a sectorectomy rather than a mastectomy [3] [4]. Furthermore the neo-adjuvant setting allows the clinical monitoring of the efficacy of the chemotherapy. An eventual pathological complete remission is a good prognostic factor although its impact on overall survival has not yet been proven [4]. Finally the frequent reticence of the patients towards chemotherapy may more easily be diminished if they can experience at least a partial remission by themselves. Adjuvant administration of the chemotherapy does not permit to profit of these clinical and psychological opportunities.

The most efficacious perioperative chemotherapy regimen is composed of two parts and three drugs. In absence of palpable axillary lymph nodes 3 cycles of docetaxel are followed by 3 cycles of an anthracyclin-cyclophosphamide combination. In presence of palpable axillary lymph nodes the same protocols are given for 4 cycles [4]. In Hungary, other protocols are currently used (e.g. FEC, $\mathrm{TAC}$ ) but these provide a lower rate of recurrence free survival and shorter overall survival [2].

Alternative regimens are proposed by the international literature as well. The neo-adjuvant treatment of triple negative tumours by the combination of docetaxel $\left(75 \mathrm{mg} / \mathrm{m}^{2}\right)$-carboplatin (AUC 6) allows a high rate of pathological complete remission [5] and the adjuvant treatment of small, node negative HER2+ tumours by the combination of paclitaxel (weekly $80 \mathrm{mg} / \mathrm{m}^{2}$ for 12 weeks)-trastuzumab (weekly $2 \mathrm{mg} / \mathrm{kg}$ for 12 weeks followed by 3 weekly $6 \mathrm{mg} / \mathrm{kg}$ for 39 weeks) allows a negligible recurrence rate together with a very good tolerance [6]. The aim of our analysis was to assess the efficacy of the two innovative alternative treatments in our patient population.

\section{Methods}

We performed a retrospective analysis of the patients treated with neo-adjuvant docetaxel-carboplatin for a triple negative, at maximum stage 
T4N3M0 primary breast cancer or with adjuvant paclitaxel-trastuzumab combination for a HER2+, at maximum stage T2aN0M0 primary breast cancer between 01.01.2012. and 31.12.2017. at the Department of Oncoradiology of the Petz Aladár Hospital, Györ, Hungary.

Every patient with a primary breast cancer corresponding to the above phenotypes and stages was eligible for the analysis. The neo-adjuvant docetaxel-carboplatin protocol was administered with a carboplatin dose of AUC 5. The adjuvant paclitaxel-trastuzumab protocol was administered with 4 to $6 \mathrm{cy}$ cles of three weekly $175 \mathrm{mg} / \mathrm{m}^{2}$ paclitaxel as compared to the original publications [5] [6].

The retrospective analysis is part of the auto-evaluation of our clinical practice. We followed validated chemotherapeutic protocols. Therefore we did not submit our study to an ethics committee approval.

For the neo-adjuvant protocol the primary outcome was the rate of pathological complete remission, the secondary outcome was the overall survival. For the adjuvant protocol the outcome was the rate of relapse.

We did not use any advanced statistical methods. Rate of pathological complete remission and relapse were calculated as proportions to the whole treated population. The survival data are given case by case in Table 1 and the median of the population was calculated.

\section{Results}

20 patients were treated with the neo-adjuvant docetaxel-carboplatin protocol (Table 1(a)). The mean age was 57.6 years (range 29 - 85 years). Pathological complete remission was noticed in 11 , a residual tumour not exceeding $10 \mathrm{~mm}$ in 4 cases respectively. 1 additional patient had partial response and 3 patients had stable disease. Progression was noticed in 1 case, for which chemotherapy was stopped and mastectomy was realised.

Regarding long term efficacy, recurrence was noticed in 5 cases. 3 patients were lost after a median follow up of 20 months, 2 patients are still receiving palliative chemotherapy. The recurring tumours were of high risk at the moment of diagnosis (stage cT4 or cN2-3). The median follow up of patients without recurrence is 41 months.

5 patients were treated with the adjuvant paclitaxel-trastuzumab protocol (Table 1(b)). The mean age was 61.5 years (range $55-75$ years). At the moment of the submission of the article all of them are free of recurrence. The median follow up was 18 months.

The decision making algorithm currently in use at our department is shown in Table 2. The algorithm corresponds to the international standards. Still it is more adapted to the phenotype of the primary tumour than the routine schemes used at many sites.

\section{Discussion}

Overtreatment by surgery and chemotherapy as well has been part of the clinical 
Table 1. Patient characteristics treated for primary breast cancer with neoadjuvant carboplatin-docetaxel (a) or adjuvans paclitaxel-trastuzumab (b). (TTR: time to recurrence from the end of the treatment, OS: overall survival from the beginning of the treatment in months, ABD: axillary block dissection, pCR: pathological complete remission, IS: in situ carcinoma.)

(a)

\begin{tabular}{|c|c|c|c|c|c|c|c|c|}
\hline Patient & Age & Tumour & Surgery & Rx therapy & Cx therapy & Residual & TTR & OS \\
\hline 1 & 39 & cT2N1, G3, mBRCA1 & Mastectomy, ABD & $50 \mathrm{~Gy}$ & 6 TXT-CBP & $4 \times 2 \mathrm{~mm}$ & - & $>7$ \\
\hline 2. & 45 & cT3N1, G3 & Mastectomy, ABD & - & 6 TXT-CBP & pCR & - & $>7$ \\
\hline 3. & 65 & cT1N2, G3 & Mastectomy, ABD & $12 \mathrm{~Gy}$ & 6 TXT-СBP & $62 \mathrm{~mm}$ & 1 & 14 \\
\hline 4. & 38 & cT2N1, G3 & Sectorectomy, ABD & - & 6 TXT-СBP & pCR & - & $>12$ \\
\hline 5. & 51 & cT2N0, G3 & Sector., sentinel & 50 Gy & 6 TXT-CBP & pCR & - & $>13$ \\
\hline 6. & 63 & cT4N2, G3 & Mastectomy, ABD & $50 \mathrm{~Gy}$ & 6 TXT-CBP & $54 \mathrm{~mm}$ & 13 & $>20$ \\
\hline 7. & 59 & cT3N1, G3 & Mastectomy, ABD & 50 Gy & 6 TXT-CBP & pCR & - & $>18$ \\
\hline 8. & 63 & cT3N1, G3 & Mastectomy, ABD & 50 Gy & 5 TXT-CBP & pCR & - & $>18$ \\
\hline 9. & 53 & cT4N2, G3 & Mastectomy, ABD & $60 \mathrm{~Gy}$ & 6 TXT-CBP & pCR & - & $>37$ \\
\hline 10. & 70 & cT1N3, G3 & Sectorectomy, ABD & $50 \mathrm{~Gy}$ & 6 TXT-CBP & pCR & - & $>41$ \\
\hline 11. & 77 & cTxN2, G3 & Mastectomy, ABD & $50 \mathrm{~Gy}$ & 6 TXT-CBP & pCR & - & $>44$ \\
\hline 12. & 85 & cT4N0, G3 & Mastectomy, ABD & $50 \mathrm{~Gy}$ & 2 TXT-CBP & $80 \mathrm{~mm}$ & 12 & 17 \\
\hline 13. & 69 & cT4N3, G2 & Mastectomy, ABD & $50 \mathrm{~Gy}$ & 5 TXT-CBP & $90 \mathrm{~mm}$ & 12 & 20 \\
\hline 14. & 55 & cT2N1, G3 & Mastectomy, ABD & - & 6 TXT-CBP & pCR & - & $>56$ \\
\hline 15. & 41 & cT2N0, G3 & Sector., sentinel & $50 \mathrm{~Gy}$ & 6 TXT-СBP & $5 \mathrm{~mm}$ & - & $>57$ \\
\hline 16. & 58 & cT4N1, G3 & Mastectomy, ABD & $60 \mathrm{~Gy}$ & 6 TXT-СBP & $27 \mathrm{~mm}$ & - & $>57$ \\
\hline 17. & 29 & cT2N0, G2 & Sector, sentinel & - & 6 TXT-СBP & $\mathrm{pCR}$ & - & $>65$ \\
\hline 18. & 61 & cT4N1, G3 & Mastectomy, ABD & - & 6 TXT-СBP & $10 \mathrm{~mm}$ IS & 35 & 36 \\
\hline 19. & 70 & cT2N1, G3 & Sector., sentinel & $50 \mathrm{~Gy}$ & 6 TXT-СBP & $3 \mathrm{~mm}$ & - & $>67$ \\
\hline 20. & 43 & cT2Nx, G3 & Sectorectomy, ABD & $50 \mathrm{~Gy}$ & 6 TXT-СBP & pCR & - & $>68$ \\
\hline
\end{tabular}

(b)

\begin{tabular}{ccccccccc}
\hline Patient & Age & Tumour & Surgery & Rx therapy & Cx therapy & Residual & TTR & OS \\
1. & 62 & pT2N0, ER- & Mastectomy, ABD & - & 4 TAX-HER & - & - & $>12$ \\
2. & 55 & pT1N1, ER-, DCIS & Sector., sentinel & 50 Gy & 6 TXT-HER & - & - & $>13$ \\
3. & 60 & pT2SN0, ER- & Sector., sentinel & 50 Gy & 6 TAX-HER & - & - & $>18$ \\
4. & 75 & pT1SN0, ER+, DCIS & Mastectomy, sent. & - & 3 TAX-HER & - & - & $>30$ \\
5. & 55 & Duplex pT2SN0, ER- & Sector., sentinel & 50 Gy & 6 TAX-HER & - & - & $>43$ \\
\hline
\end{tabular}

Table 2. Petz algorithm for the perioperative treatment of primary breast cancers. (A: adriamycin, C: cyclophosphamide, T: docetaxel, TXT: docetaxel, TAX: paclitaxel, HER: trastuzumab, PER: pertuzumab, CBP: carboplatin. Adriamycin may be replaced by dose equivalent epirubicin.)

\begin{tabular}{ccccc}
\hline & Luminal A & Luminal B & HER2+ & Triple negative \\
\hline Neo-adjuvant & Hormonal & AC + T & AC + T-HER-(PER) & TXT-CBP \\
Adjuvant & AC $+\mathrm{T}$ & AC $+\mathrm{T}$ & TAX-HER & AC + T
\end{tabular}


practice. The possibility to obtain the same recurrence free rate by less aggressive interventions was first proven in the field of surgery. The Halstead amputations were gradually replaced by simple mastectomy and when possible sectorectomy as well as the axillary block dissections by sentinel techniques and radiotherapy [3]. There are recent publications dealing with the possible reduction of the undesirable sequelae of sentinel node surgery [7]. Similarly, there are ongoing attempts to avoid the overtreatment by chemotherapy. One of these attempts, the estimation of the recurrence risk by multigene array has become available in several countries [4]. Another attempt is the replacement of the considerably toxic triple regimen for selected patients [5] [6].

The neo-adjuvant docetaxel-carboplatin protocol was originally described with a carboplatin dose of AUC 6 [5]. We used a carboplatin dose of AUC 5. The adjuvant paclitaxel-trastuzumab protocol was described with 4 cycles of weekly paclitaxel [6]. We used 4 to 6 cycles of three weekly paclitaxel. Thus our protocols were less toxic and easier to schedule. No major toxicity was noticed with these protocols. Granulocyte colony stimulating factor (GCSF) profilaxis was systematically added to the docetaxel-carboplatin protocol.

The cohort of 20 patients who received the docetaxel-carboplatin combination can be considered as statistically representative. The good pathological complete remission and survival data show the value of the alternative chemotherapy for these tumours of especially unfavourable prognostic (of high grade and refractory to hormone- and growth factor receptor targeted treatment). The cohort of 5 patients who received the paclitaxel-trastuzumab combination is statistically too small. The results are considered as advertising rather than approving. (The protocol does not figure in the Hungarian Textbook for Chemotherapy [8] and up to our knowledge it is not widely used in our country, therefore we believe the publication of the data is worthwhile.)

There is no sufficient evidence to support the role of adjuvant radiotherapy in case of pathological complete remission obtained with chemotherapy. Initial tumour extension and nodal involvement may influence the decision [9]. Two patients did not receive adjuvant irradiation of our decision, three other patients of their own decision.

Trastuzumab can be used in neo-adjuvant setting as well for HER2+ tumours and it allows a high rate of pathological complete remission. The addition of pertuzumab to the combination may further rise the rate of pathological complete remission but it has not been proven to have an impact on the overall survival, thus its systematic use is not proposed [3] [4].

According to novel results axillary block dissection may be replaced by radiotherapy in cases when only microscopic metastases are found in the sentinel lymph nodes [3]. If it comes confirmed that radiotherapy can eradicate the clinically undetectable tumours then the treatment of primary breast cancers showing complete remission after neo-adjuvant chemotherapy could be limited to chemotherapy and radiotherapy alone, permitting to avoid surgery for selected patients. 
Immuno-radiotherapy is subject of investigations. According to some hypotheses the application of neo-adjuvant irradiation and checkpoint inhibition may have a synergetic action by the parallel release of tumour antigens and activation of the immune system [10].

Dose dense adjuvant chemotherapy is another field of investigation. The density may be raised by the increase of the treatment doses or the decrease of the intervals between two treatments. Both strategies improve the recurrence free rate and the overall survival by some percents on the expense of higher toxicity [10].

The molecular and immunologic characterisation of triple negative tumours is proposed to yield more specific and hopefully efficacious targeted treatments for this still poor prognostic patient population [11].

The optimal duration of adjuvant chemotherapy for HER2+ breast cancers is debated. Although the non-inferiority of a 9 months long treatment was not approved in an earlier study [10], the 6 months long treatment allowed similar survival data as the standard 12 months treatment according to a recent publication [12].

The main limitation of our study is the relatively small number of patients included. As the original international studies were large trials, they stand for the validity of our results. We believe that the interest of our work is the flexible use of different upcoming literature data that allow a phenotype dependent management of our patients with primary breast cancer. In addition, these protocols are less toxic or more comfortable to schedule as compared to the standard regimens.

\section{Conclusions}

The neo-adjuvant docetaxel-carboplatin combination may allow a good rate of pathological complete remission for triple negative breast cancers.

The adjuvant paclitaxel-trastuzumab combination may allow a good recurrence free rate for HER2+, node negative breast cancers of small size.

\section{Conflicts of Interest}

The authors declare no conflicts of interest regarding the publication of this paper.

\section{References}

[1] Kásler, M., Ottó, S. and Kenessey, I. (2017) The Current Situation of Cancer Morbidity and Mortality in the Light of the National Cancer Registry. Hungarian Medical Journal, 158, 84-89.

[2] Újhelyi, M., Pukancsik, D., Kelemen, P., Sávolt, Á., Gődény, M., Kovács, E., Udvarhelyi, N., Bak, M., Polgár, C., Rubovszky, G., Kásler, M. and Mátrai, Z. (2016) Breast Cancer Care Quality Analysis of the National Institute of Oncology in Hungary According to the Requirements of European Society of Breast Cancer Specialists (EUSOMA). Hungarian Medical Journal, 157, 1674-1682.

[3] ESMO Clinical Practice Guidelines. 
http://www.esmo.org/Guidelines/Breast-Cancer/Primary-Breast-Cancer

[4] Horváth, Zs., Boér, K., Dank, M., Kahán, Zs., Kocsis, J., Kövér, E., Pajkos, G., Pikó, B., Rubovszky, G. and Eckhardt, S. (2016) Systemic Treatment of Breast Cancer: Professional Guidelines. Hungarian Oncology, 60, 241-257.

[5] Sharma, P., López-Tarruella, S., García-Saenz, J.A., et al. (2017) Efficacy of Neoadjuvant Carboplatin Plus Docetaxel in Triple-Negative Breast Cancer: Combined Analysis of Two Cohorts. Clinical Cancer Research, 23, 649-657. https://doi.org/10.1158/1078-0432.CCR-16-0162

[6] Tolaney, S.M., Barry, W.T., Dang, C.T., et al. (2015) Adjuvant Paclitaxel and Trastuzumab for Node-Negative, HER2-Positive Breast Cancer. New England Journal of Medicine, 372, 134-141. https://doi.org/10.1056/NEJMoa1406281

[7] Kozak, D., Głowacka-Mrotek, I., Nowikiewicz, T., Siedlecki, Z., Hagner, W., Sowa, M. and Zegarski, W. (2018) Analysis of Undesirable Sequelae of Sentinel Node Surgery in Breast Cancer Patients: A Prospective Cohort Study. Pathology \& Oncology Research, 24, 891-897. https://doi.org/10.1007/s12253-017-0306-3

[8] Hungarian Textbook for Chemotherapy http://www.neak.gov.hu/felso_menu/szakmai_oldalak/gyogyito_megeleozo_ellatas/ szabalykonyvek/kemo_prot.html 01.12.2018.

[9] Kishan, A.U. and McCloskey, S.A. (2016) Postmastectomy Radiation Therapy after Neoadjuvant Chemotherapy: Review and Interpretation of Available Data. Therapeutic Advances in Medical Oncology, 8, 85-97. https://doi.org/10.1177/1758834015617459

[10] Kahán, Zs. (2018) Novelties in the Management of Breast Cancer in the Light of the San Antonio Breast Cancer Symposium 2017. Clinical Oncology, 5, 11-15.

[11] Gregório, A.C., Lacerda, M., Figueiredo, P., Simões, S., Dias, S. and Moreira, J.N. (2018) Therapeutic Implications of the Molecular and Immune Landscape of Triple-Negative Breast Cancer. Pathology \& Oncology Research, 24, 701-716. https://doi.org/10.1007/s12253-017-0307-2

[12] Earl, H.M., Hiller, L., Vallier, A.L., et al. (2018) PERSEPHONE: 6 versus 12 Months of Adjuvant Trastuzumab in Patients with HER2 Positive Early Breast Cancer: Randomised Phase 3 Non-Inferiority Trial with Definitive 4-Year Disease-Free Survival Results. http://abstracts.asco.org/214/AbstView_214_217191.html 